\title{
Magnetic and Electric Charges in Physics and Technology of Electromagnetic and Magnetoelectric Induction
}

\author{
Robert A. Sizov* \\ Solid State Physics Institute, Moscow Region, Russia \\ Email: sizov.robert@gmail.com
}

How to cite this paper: Sizov, R.A. (2017) Magnetic and Electric Charges in Physics and Technology of Electromagnetic and Magnetoelectric Induction. Journal of Modern Physics, 8, 964-981.

https://doi.org/10.4236/jmp.2017.86061

Received: April 27, 2017

Accepted: May 28, 2017

Published: May 31, 2017

Copyright $\odot 2017$ by author and Scientific Research Publishing Inc. This work is licensed under the Creative Commons Attribution International License (CC BY 4.0).

http://creativecommons.org/licenses/by/4.0/ (c) (i) Open Access

\begin{abstract}
Experimental and theoretical researches performed by the author (period: 1969-present) showed that magnetic spinor particles (magnetic charges) are real structural components of atoms and substance and immediate sources of all magnetic fields and magnetic manifestations in Nature. Magnetic charges, that constitute together with electrical charges atomic shells got of title: magnetons and antimagnetons (respectively with charges $\mathrm{g}^{-}$and $\mathrm{g}^{+}$under fundamental condition: $g=e$ ). Furthermore, in addition to participating in structures of atomic shells, the magnetic charges exist in potential and even in real zones conduction of solids. The magnetic and electric spinor particles in atoms and substance exist in form such of the spinor associations as the magnetic and electric bispinors. Under influence of external magnetic field in conductor being implemented polarization of magnetic bispinors with formation of magnetic dipoles. Internal fields magnetic dipoles is directed against an external magnetic field and is a simple explanation of such a physical manifestation as diamagnetism. During the rotation of these dipoles in the conductor are formed of the vortex magnetic dipole fields, which and create an electromotive force, i.e. are responsible for the electromagnetic induction. Author proposed new magneto-electric technology called the magnetoelectric induction, as the result of which the permanent current of magnetic charges is created. However, such currents may be implemented solely in the superconductors. In the article presented the main reasons which more hundred year old hinder recognition and technical use of the real magnetic charges and their currents what considerably slow down the global scientific and technological progress.
\end{abstract}

\section{Keywords}

Magnetic Spinor Particles (Spinors and Antispinors), Magnetons and 
Antimagnetons, Bispinors, Vortex Spinor Fields, Electromagnetic and Magnetoelectric Induction

\section{Magnetic Spinor Particles (Magnetic Charges) Are Immediate Sources of All Magnetic Fields in Nature}

Magnetic charges (magnetic spinor particles) which are structural elements of atoms and substance, as well as by the immediate sources of all magnetic fields in the Nature, were discovered by the author and were first presented to scientific society in 2001 in publication [1]. Circumstances that prompted the author to study problem participation of real magnetic charges in structures of atoms and substance were his experiments with the magnetic neutron scattering in ferrimagnetic crystals [2] [3] (1970-1971). Discovery in these experiments of significant displacements of all density so-called magnetic moments $\mathrm{Fe}^{3+}$ ions from their nuclei served as a basis for the author's assumption of possibility structural participation of the real magnetic spinor particles in atoms and substance. It is important to note that as result of the above-mentioned displacements of the so-called magnetic moments in crystallattice of ferrimagnet is realized new type of magnetic ordering: the helicoidal chain magnetic structure.

The results of numerous subsequent experiments conducted by the author showed that in structures of Physical mass (atoms, nucleons, substance, etc.) the magnetic particles (spinors and antispinors) are present, approximately, in same amount as the electric particles. Detailed description experiments of author on the problem of the real magnetic charges is given in publications [4] [5], and their summaries can be found in [6].

Magnetic spinors within atomic shells (the author calls them magnetons) are fundamental material particles which by their physical parameters are magnetic analogs of electrons, i.e. they have a values of charges, spins and measures of inertia equal to electrons. Magnetons, like electrons, exhibit a charge with negative sign (g-), they relate to the class of leptons, and on its statistic properties to fermions (the spin equal to $1 / 2$ ).

Note 1 . The magneton name which was given of the material magnetic particle, i.e. for the magnetic spinor, which is included as a structural component in shells of atoms, is according to the author best possible solution (see: electronmagneton). This magnetic spinor particle is magnetic analog of electron. Latter name is designed to preserve very important terminological symmetry in physical science.

As for such well-established concept and term as the Bohr magneton, which is unit of the theoretical magnetic moment, its occurrence is associated exclusively with "absence" in physical representations of real magnetic charges. Introduction in physical representations of real magnetic poles (charges) as the constituent of atoms and substance is making the tangled notions of magnetic moment completely superfluous.

Magnetic antispinors, for example, the antimagnetons, are true antiparticles 
in relation to magnetons. They have a positive magnetic charge $\left(\mathrm{g}^{+}\right)$and relate to antileptons. By their statistic properties the antimagnetons are antifermions with an antispin $(-1 / 2)$ in relation to spinors.

Note 2. For greater lucidity, we note that the concept "the spinor particle" used in the article should be attributed solely to fundamental particle with electric or magnetic charge, and term the "the spinor particle" is synonymous of term "the charged particle".

The magnetic bispinor, i.e. conjoint magnetic spinor pair: magneton and corresponding its antimagneton, under rotation in a lattice of conductor will be create in plane of rotation of well-known the vortex magnetic field, which is determined by the axial vector rotH ${ }^{\circ}$. Process formation of latter field is described by equation: $\mathrm{k}_{2} \operatorname{rot} \mathbf{J}_{\mathrm{g}}^{\mathrm{o}}=\operatorname{rot} \mathbf{H}^{\mathrm{o}}$, where $\operatorname{rot} \mathbf{J}_{\mathrm{g}}^{\mathrm{o}}$ vector of vortex current of magnetic charges, and in model representation is the rotating the polarized magnetic bispinor or magnetic spinor dipole, $\mathrm{k}_{2}$-the proportionality factor.

Role of electrical current in this process consists solely in "organizing" of vortex (circular) motion of magnetic spinordipoles. Process formation the vortex current of magnetic charges is described by equation: $k_{1} \mathbf{J}_{\mathrm{e}}=\operatorname{rot} \mathbf{J}_{\mathrm{g}}{ }_{\mathrm{g}}$, where $\mathrm{J}_{\mathrm{e}}$ is vector of density linear electric current. Coefficients $k_{1}$ and $k_{2}$ in equations mentioned above, referring to different physical processes which are consistently implemented in a conductor under conditions of the experiment Oersted and, in principle, not equal to each other, i.e. $\mathrm{k}_{1} \neq \mathrm{k}_{2}$. Physical meaning of small circles with vectors of strength of fields and currents, as well as a systematic of electric and magnetic bispinor fields are given below in Section 2.

Thus, sequence of real physical processes in conductor with a constant electric current, which leads to formation of the vortex magnetic field around conductor can be written as:

$$
\mathbf{J}_{\mathrm{e}} \rightarrow \operatorname{rot} \mathbf{J}_{\mathrm{g}}^{\mathrm{o}} \rightarrow \operatorname{rot} \mathbf{H}^{0} .
$$

It is important to note once again that latter sequence is the physically precise of mathematical designation of processes that make up the famous experiment of Oersted. Accuracy of description here in the fact, that in this sequence are included the real sources of magnetic field, i.e. the magnetic poles or magnetic charges.

We will notice that in Maxwell's electromagnetic (EM) concept in which real magnetic charges were ignored (unintentionally), sequence of processes at formation of the vortex magnetic field round the conductor with electric current is represented as follows: $\mathbf{J}_{\mathrm{e}} \rightarrow \operatorname{rot} \mathbf{H}^{\circ}$ [7]. Last sequence, which corresponds to a first Maxwell equation $\left(\mathrm{k} \mathbf{J}_{\mathrm{e}}=\operatorname{rot} \mathbf{H}^{\circ}\right.$ ), was the result of superficial glance of Maxwell on the Oersted's experiment, in which were taken into account only the external, i.e. explicit components of process: the electric current and magnetic field around conductor. The "thanks" to this (superficial) look of Great physicist in of electromagnetic theory, more than on 100 years old, were "alive buried" the immediate sources of magnetic field, i.e. the magnetic poles or magnetic charges.

Note 3. Detection and investigations of magnetic charges in structures of 
atomic shells that are managed to make the author and which formed basis for his fundamental conclusions, should not be seen as something unique. Merit of the author is only in the fact that he showed participation of real magnetic charges in structures of atoms, substance and, in general, in compositions of the Physical Mass. Of course, this is a very important conclusion. This was followed by chain of original assumptions and experiments that, in the end and led author to his Gravito-Physics and Gravito-Technics [8] [9] [10] [11]. As for fact of first detection of real magnetic charges, then it is a merit, first of all, a wonderful Felix Ehrenhaft, who made his discoveries in the early 20th century and his numerous followers [12]. The reasons, which more than hundred years, block the recognition of fundamental experiments and informed conclusions $\mathrm{F}$. Ehrenhaft, discussed in detail in the publications of the author [1] [6] [13].

It should be noted that aim of researches in this work is interpretation of the physics of electromagnetic induction with the inclusion in of views of real magnetic charges discovered by the author in the substance. In addition, it was supposed to detect possible new electromagnetic technologies that can carry with them true of magnetic poles. As shown in this article, namely by such a new technology can be of the magnetoelectric induction.

\section{Magnetic and Electric Bispinors in Substance and Modifications of Spinor Fields in Nature}

Detection by the author of real magnetic charges in atoms and substance, as well as introduction in physical representations of such stable associations of spinor particles as the magnetic and electric bispinors, require revision of the generally accepted in Physics of systematization spinor fields. It is extremely important to note that association and stable stay of spinor particles in compositions of bispinors are their fundamental property.

Namely in compositions of bispinors the spinor particles are realized as in atoms, so and in substance. As for atomic bispinors, which forming of electromagnetic shells of atoms, so this topichas been repeatedly reviewed in numerous publications by the author [1] [5] [6] [14]. The spinor particles considered in this article and which are involved in processes of electromagnetic and magnetoelectric inductions are inhabited in conductors in real or potential zones of conductivity.

The magnetic and electric bispinors in absence of external influences are in the state of so-called the spin-short. This condition is characterized by densely pressing the spinor and antispinora each to other, what is accompanied by compensation of activity their spins. In this state spinor pairs as if disappearing and not manifest itself by spinor fields. Such a spinor pairs can also be called as closed or spin-shorted bispinor. The equation of state spin-shorted spinor pair can be represented as follows: $S_{\mathrm{sp}}+\left(-\mathbf{S}_{\mathrm{asp}}\right)=0$, where $\mathbf{S}_{\mathrm{sp}}$ and $\mathbf{S}_{\mathrm{sp}}$ are the spin vectors of the spinor and antispinor.

It is important to stress that aconditions of spin-shorting in spinor pairs, i.e. in bispinors, it does not have relationship to the annihilation of par- 
ticles, as this is commonly believed. Annihilation processes take place in electron-positron pairs, but this is a completely different story, since the positron is not a true antielectron. In his publications, the author showed that positron, with the high degree of probability, is a subject of mass, since it, unlike the electron, participates in the gravitational interaction. The true antielectron "sits" in the positron structure and it is he who determines the electric charge $\left(\mathrm{e}^{+}\right)$of the positron. Upon contact of electron with positron is association of electron and true antielectron with the formation of spin-shorted bispinor, and all excess of fine particles is thrown away. Evidence of the destruction a structure of pozitronupon its contact with an electron is gamma quanta. In this, in fact, and consists known of process annihilation of particles.

Moreover, the spin-shorted state is the natural state of spinor particles what is corresponds of principle of minimizing "Dark energy" or by principle of least action. More information about the spin-shorting of particles, the minimizing of "Dark energy" and "annihilation" of particles is given in the author's publications [5] [6] [13].

Thus, according to the results of author's research, bispinors as both electric and magnetic may reside in two states: 1) the state of closed or spin-shorted of bispinor (basic or unperturbation it state);2) the polarized state or state of spinor dipole.

Under influence of external spinor fields there occurs a violation of the spin-short state of bispinors with a formation of spinor dipoles. The scheme for the formation of magnetic spinor dipoles in a conductor under the action of an external magnetic field is shown in Figure 1. Here, the small circles denote the magnetic spinors (magnetons) and corresponding of them the magnetic antispinors (antimagnetons). Large circles denote atoms in the conductor lattice. Vectors $\mathbf{H}_{\mathrm{bs}}$ of the strength of internal static fields of magnetic dipoles are directed against external field, what is simple explanation of such a physical manifestation as diamagnetism. Subsequently, we will determine the internal spinor fields formed in the framework of polarized bispinors or spinor dipoles, as the bispinor fields.

Subsequently, we will determine the internal spinor fields formed in framework of polarized bispinors, i.e. spinor dipoles, as the bispinor fields. Vectors $\mathrm{H}_{\mathrm{bs}}$ of the strength of internal static fields of magnetic dipoles are directed against external field, what is simple explanation of such a physical manifestation as diamagnetism. Subsequently, we will determine the internal spinor fields formed in the framework of polarized bispinors or spinor dipoles, as the bispinor fields.

The magnetic and electric spinor dipoles, i.e. polarized bispinors, form in the plane of their rotation the vortex spinor fields, which in modern theories are determined by the vortex vectors rotH and rotE. It is important to note that vectors $\mathrm{H}$ and $\mathrm{E}$ strength of fields, standing under the sign of the operator rot, are by vectors instantaneous strength of respective vortex fields. These vectors should be denoted as $\mathbf{H}^{\circ}$ and $\mathbf{E}^{\circ}$. Small scircles with vectors of strength a fields determine to the instantaneous or dynamic strength of the vortex fields, and the divergence of such vectors is equal to zero $\left(\operatorname{div} \mathrm{E}^{\circ}=0\right.$ and $\left.\operatorname{div} \mathrm{H}^{\circ}=0\right)$. 


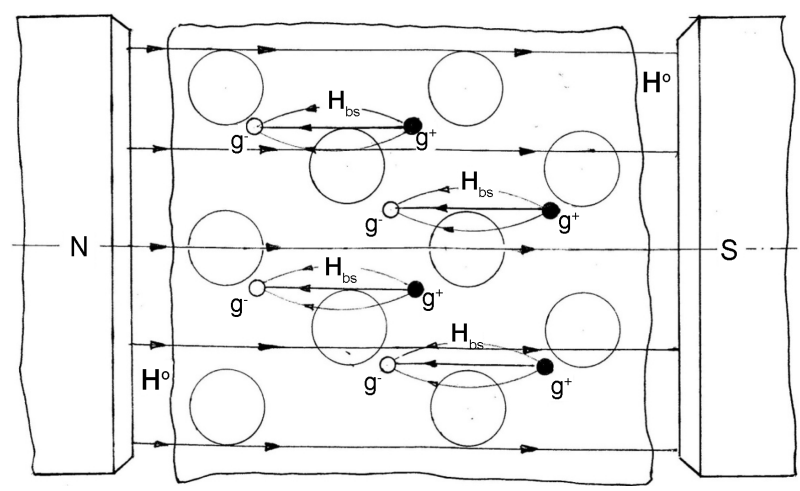

Figure 1. Scheme of polarization magnetic bispinors in lattice of conductor when influence of external magnetic field.

It is important to understand that all stable vortex spinor fields in Nature are formed by rotationing of spinordipols, both magnetic so and electrical and such fields should be called as of vortex bispinor fields (VBF).

Depending on the rotational speed of bispinors, VBF are divided into two types: the "fast" fields and "slow" fields. As will be shown in the article, these fields are decisive in processes induction a currents of charges within the framework of electromagnetic and magnetoelectric induction.

Quotes with words "fast" and "slow" emphasize that these definitions refer exclusively to the motions of bispinors, which form the vortex fields, but not relate to the velocitie of propagation of fields in space. In the following we omit the quotation marks, keeping in mind the above meanings of words: fast and slow.

Fast fields are formed by rotating of spinor dipoles, which are untwist in the lattice of conductor by charges currents. The circumferential velocities of the particles in such processes are comparable with speed of light. An example of such fields is magnetic field, which is formed around conductor with a constant electric current. Model representations of processes formation of such fields is given below in Section 3 .

As for the slow vortex bispinor fields, they are formed by spinor dipoles, the rotations of which is effected by rotating of the conductive frame in an external field or as the result of rotation of the poles $\mathrm{N}$ and $\mathrm{S}$ of the source of the external field. The strength of these slow bispinor fields will be denoted as $\mathbf{H}^{\circ}{ }_{\mathrm{sl}}$ and $\mathbf{H}_{\mathrm{sl}}^{\mathrm{o}}$ where "sl" from slow.

To the aforementioned bispinor fields one should add of static bispinor fields, which are formed within the framework of static bispinors (see Figure 1). The strength of these fields in our paper denoted as $\mathrm{H}_{\mathrm{bs}}$ (for magnetic bispinor fields) and $\mathbf{E}_{\mathrm{bs}}$ (for electric bispinor fields). Given that the bispinor fields are closed and realized within the framework of a pair of related charges of the opposite sign, the divergence of the vectors $\mathrm{H}_{\mathrm{bs}}$ and $\mathrm{E}_{\mathrm{bs}}$ will be equal to zero ( $\operatorname{divH}_{\mathrm{bs}}=0$ and $\operatorname{divE}_{\mathrm{bs}}=0$ ).

The Individual spinor particles, which are not included in the bispinor system, emit the spinor fields the strength of which is determined by the polar vectors $\mathrm{H}$ and $\mathbf{E}$ (without small circles). The divergence of such vectors, as is known, is de- 
fined as: $\operatorname{divH}=4 \pi \rho_{\mathrm{g}}$ and $\operatorname{divE}=4 \pi \rho_{\mathrm{e}}$, where $\rho_{\mathrm{g}}$ and $\rho_{\mathrm{e}}$ are the density, respectively, magnetic $(\mathrm{g})$ and electric (e) charges.

Note that small circles used in the notations of vectors vortex fields must also use and in notations vectors density of vortex currents electric and magnetic charges, for example, $\mathbf{J}_{\mathrm{e}}^{\mathrm{o}}$ and $\mathbf{J}_{\mathrm{g}}^{\circ}$. It should be added that the last vortex currents form fast bispinor fields, i.e. fields determined by the vortex vectors $\operatorname{rot}^{\circ}$ and rotH $^{\circ}$. As for slow VBF, so they are formed by slow eddy currents, i.e. slowly rotating bispinors and are respectively denoted by $\mathbf{J}_{\text {e(sl) }}^{\circ}$ and $\mathbf{J}_{\mathrm{g}(\mathrm{sl})}^{\mathrm{o}}$.

We will name the main reasons that led to the existing confusion in the representations, and consequently and in the notation of spinor fields.

1) Ignoring in Physics science, beginning with Maxwell, of real magnetic charges, as well as true antielectrons (but not positrons), which as it does seem paradoxical, are real structural components of atoms and substance.

2) Lack of stable natural sources of vortex electric field that is generated by the rotating electrical bispinors (spinor electric dipoles) and which describes by the vortex vector rotE $\mathrm{E}^{\circ}$. Equation education of last field has the form:

$\mathrm{k}_{2} \operatorname{rot} \mathbf{J}_{\mathrm{e}}^{0}=\operatorname{rot} \mathbf{E}^{0}$.

The sequence of physical processes in conductor (more precisely, in a superconductor), which leads to a formation of vortex electric field, which is described by vortex vector $\operatorname{rot} \mathrm{E}^{\circ}$, is as follows:

$$
\mathbf{J}_{\mathrm{g}} \rightarrow \operatorname{rot} \mathbf{J}_{\mathrm{e}}^{\mathrm{o}} \rightarrow \operatorname{rot} \mathbf{E}^{\mathrm{o}}
$$

where $\mathrm{J}_{\mathrm{g}}$ is vector a density of linear current of magnetic charges, and $\operatorname{rot}^{\circ}{ }_{\mathrm{e}}{ }_{\text {is }}$ vector of vortex current of electric charges.

In all its publications, starting in 2001 [1], the author has consistently emphasized, that main reasons for the fact that real magnetic charges were "alive buried" in the modern theories is Physics their confinement in atoms and substance which cardinal differently from confinement, for example, of electrons.

Namely, the features of Physics of confinement of magnetic charges in the substance, were the reason for the formation of the fallacious concept of Maxwell's electromagnetism in the framework of which, really existing a magnetic poles, have been mistakenly substituted on acts of mechanical movement of electrical charges. Read more the problem of confinement of electric and magnetic spinor particles in of atoms and substance, is given in the publications of the author [6] [13], and summarized in section 5 of this article.

\section{Magnetic and Electric Bispinors in Processes Formation VBF Determining by Vortex Vectors $\operatorname{rot}^{\circ}$ and $\operatorname{rot}^{\circ}$ (Model Representation)}

Considera processes education of vortex magnetic and electric fields, which are determined by vortex vectors $\operatorname{rot}^{\circ}$ and $\operatorname{rotE}^{\circ}$. Figure 2 shows scheme processes formation of vortex fields: magnetic, corresponding to the equation

$$
\mathrm{k}_{2} \operatorname{rot} \mathbf{J}_{\mathrm{g}}^{\mathrm{o}}=\operatorname{rot} \mathbf{H}^{\mathrm{o}}
$$

and electric 


$$
\text { (equation } \mathrm{k}_{2} \operatorname{rot} \mathbf{J}_{\mathrm{e}}^{\mathrm{o}}=\operatorname{rot} \mathbf{E}^{\mathrm{o}} \text { ). }
$$

As noted above vortex vectors $\operatorname{rot}_{\mathrm{g}}^{\mathrm{o}}$ and $\operatorname{rot}^{\mathrm{o}}{ }_{\mathrm{e}}$, representing respectively the vortex (circular) currents of magnetic and electrical charges, in reality, are rotating magnetic and electric spinor dipoles.

As shown by the author in his publications [15] [16], antielectrons, which together with electrons form spinor pairs (electric bispinors), are real particles of Antimatter and are neither positrons, neither "holes" Dirac [17]. Recall that the values of charges spinor particles involved in the processes of electromagnetic and magnetoelectric induction, determined by a condition $\mathrm{g}=\mathrm{e}$.
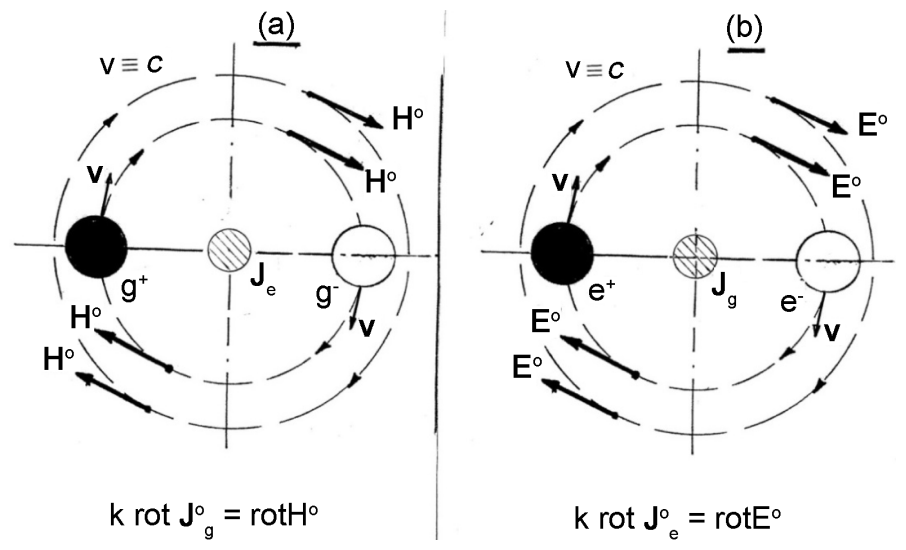

Figure 2. Schemes for formation of VBF by rotating bispinors: magnetic (Figure 2(a)) and electric (Figure 2(b)). The magnetic bispinor (see Figure 2(a)) consists of magnetic spinor, i.e. Magneton (white circle) and magnetic antispinor is antimagneton (black circle), respectively with charges $\mathrm{g}^{-}$and $\mathrm{g}^{+}$. The electric bispinor (see Figure $2(\mathrm{~b})$ ) consists of electric spinoris electron (black circle) and electrical antispinor i.e. antielectron (black circle) with charges $\mathrm{e}^{-}$and $\mathrm{e}^{+}$.

Linear currents of electric $\left(\mathrm{J}_{\mathrm{e}}\right)$ and magnetic $\left(\mathrm{J}_{\mathrm{g}}\right)$ charges, that induce a vortex motion, respectively, of magnetic and electric bispinors, are normal to the plane of Figure 2 and indicated by hatched circles. It is important to understand that these linear currents are only intermediaries, which deduce a spinor pairs (bispinors) from a state of spin-shorting and lead the magnetic and electric dipoles into a state of rotational movement.

It is also important to note that the formation of vortex bispinor fields which are shown in Figure 2, possible under a condition: $\mathrm{V} \equiv \mathrm{c}$, where $\mathrm{c}$ is the speed of light. As were agreed noted above in Section 2 the vortex bispinor fields, rot $\mathrm{H}^{\circ}$ and $\operatorname{rot} \mathrm{E}^{\circ}$ refer to the so-called fast vortex fields.

\section{Magnetic and Electric Charges in Physics and Technology of Electromagnetic and Magnetoelectric Induction}

The complex of physical processes in result of which is obtained of linear current of the electric charges $\left(\mathrm{J}_{\mathrm{e}}\right)$ in the conductor is called the electromagnetic induction (EMI). In general view a sequence of processes in this case has the form: $\operatorname{rot} \mathbf{H}^{\mathrm{o}} \rightarrow \operatorname{rot} \mathbf{J}_{\mathrm{g}}^{\mathrm{o}} \rightarrow \operatorname{rot} \mathbf{H}_{\mathrm{sl}}^{\mathrm{o}} \rightarrow \mathbf{J}_{\mathrm{e}}$. 
It should be noted that in frame work of the first stage of this sequence, i.e. $\operatorname{rot} \mathbf{H}^{\circ} \rightarrow \operatorname{rot} \mathbf{J}_{\mathrm{g}}^{\mathrm{o}}$, in conductor are implemented by two physically different processes: 1) polarization of magnetic bispinors in conductor under influence of the external magnetic field with formation of static magnetic dipoles; 2) implementation of rotational motion of these dipoles, for example, through rotation of the conducting frame in a magnetic field.

Physics of educationstatic of static magnetic dipols and their fields with strength $\mathbf{H}_{\mathrm{bs}}$ within a substance under the influence of an external magnetic field has been considered above in section 2 (see Figure 1).

Provision of rotational motion magnetic dipoles in conductor under influence of external magnetic field, it is the most important stage in the electromagnetic induction. For this in the existing electrotechnique is used two ways:

1) Rotation of conducting frame in the magnetic field;

2) Rotation of magnetic field, i.e. rotation of poles $\mathrm{S}$ and $\mathrm{N}$ of magnet around of linear conductor.

The sequences of processes, in result which in conductor are formed rotating magnetic spinor dipoles, for each of the ways mentioned above, can written in form: 1) $\operatorname{rot} \mathbf{H}^{\mathrm{o}} \rightarrow \operatorname{rotrot} \mathbf{J}_{\mathrm{g}(\mathrm{sl})}^{\mathrm{o}}$;2) $\operatorname{rotrot} \mathbf{H}^{\mathrm{o}} \rightarrow \operatorname{rot} \mathbf{J}_{\mathrm{g}(\mathrm{sl})}^{\mathrm{o}}$. Expression $\operatorname{rotrot} \mathrm{J}_{\mathrm{g}(\mathrm{s} \mathrm{l})}^{\mathrm{o}}$ corresponds to process of rotation of the conducting frame, and expression rotrot $\mathrm{H}^{\circ}$ corresponds to rotation around the linear conductor of magnetic poles of the $\mathrm{N}$ and $\mathrm{S}$. It is to note that the rotation of the frame and the magnetic poles is carried out by external forces (by electric motor), which is emphasized in the article by the operator rot.

The next stage of EMI is formation of vortex fields of magnetic dipoles, which are described by vortex vector rotH $^{\circ}{ }_{\mathrm{sl}}$ and which are implemented under rotational motion of magnetic dipoles according to the equation:

$\mathrm{k}_{2} \operatorname{rot} \mathbf{J}_{\mathrm{g}(\mathrm{sl})}^{\mathrm{o}}=\operatorname{rot} \mathbf{H}_{\mathrm{sl}}^{\mathrm{o}}$. It is important to note that the vortex vector $\mathrm{H}_{\mathrm{sl}}^{\mathrm{o}}$ determines the strength of slow VBF, and in contrast to the polar vector $\mathrm{H}_{\mathrm{bs}}$ (see Figure 1), is the instantaneous vector of strength.

The final stage of EMI is in that under the influence of the vortex bispinor field $\left(\operatorname{rotH}_{\mathrm{sl}}^{\mathrm{o}}\right)$ is induced the electric current. The equation of the latter process is as follows: $\mathrm{k}_{3} \operatorname{rot} \mathbf{H}_{\mathrm{sl}}^{\mathrm{o}}=\mathbf{J}_{\mathrm{e}}$. It is important to recall, that the k-coefficients $\left(k_{1}, k_{2}, k_{3}\right)$ in equations that are given in the article, in principle, not equal to each other.

Turning to the technical stages, corresponding to magnetoelectric induction (MEI), it should be noted that they are all magnetoelectric counterparts of processes that make up the electromagnetic induction. The difference here is in special conditions confinement of electrons and magnetic charges in substance. The essence these differences are listed in section 5. These distinctions are creating, for example, such a limitation in implementation of processes of magnetoelectric induction, as the mandatory use of superconductors.

In addition, a significant obstacle to the receipt of current the magnetic charges within the MEI, is a lack in the Nature of the stable source of vortex electric field, which is defined by the vortex vector $\operatorname{rot}^{\circ}$. The last field is an 
electrical analog of the vortex magnetic field, described by the vortex vector $\operatorname{rot}^{\circ}$. Should also be noted that the lack of stable fields the $\operatorname{rot}^{\circ}$ in Nature, again, associated with the differences in Physics of confinement the magnetic and electrical charges in substance. Note also that creating sources of stable fields $\operatorname{rot}^{\circ}$, is a serious technical problem. It is also important to emphasize that all known ferroelectrics are not sources of the vortex electric field, i.e. they are not electrical analogs of ferromagnetics.

Thus, if we have in the arsenal of tools are the superconductors and stable sources of vortex electric field $\left(\operatorname{rot}^{\circ}\right)$ then without special problems it is possible to organize the processes of magnetoelectric induction and receive the stable currents of magnetic charges.

The sequence of processes defining MEI, as a result of which we obtain a linear magnetic charges current $\left(\mathrm{J}_{\mathrm{g}}\right)$ in a conductor (or more precisely, in a superconductor), has the form: $\operatorname{rot} \mathbf{E}^{0} \rightarrow \operatorname{rot} \mathbf{J}_{\mathrm{e}(\mathrm{sl})}^{0} \rightarrow \operatorname{rot} \mathbf{E}_{\mathrm{sl}}^{0} \rightarrow \mathbf{J}_{\mathrm{g}}$. Since this technology is brand new, we consider in the detail of its stages.

When exposed of external vortex electric field $\left(\operatorname{rot}^{\circ}\right)$, in superconductor is formed of electric spinor dipoles like form magnetic dipols, which are realized in conductor when exposed of external magnetic field $\left(\operatorname{rot}^{\circ}\right)$. When a rotating of superconducting frame in an external electric field $\left(\operatorname{rot}^{\circ}\right)$ or when rotating of poles $\mathrm{S}$ and $\mathrm{N}$ of source such a field around of the superconductor, in it forms a system of rotating electrical dipoles, i.e. of circular electric currents, determined by vortex vector $\operatorname{rot}^{\mathrm{o}}{ }_{\mathrm{e}(\mathrm{s})}$. The similar effect is implemented and in the EMI, but with the magnetic spinor dipoles.

The penultimate stage, which is implemented in framework of magneto-electric induction is the formation of vortex field $\left(\operatorname{rotE}_{\mathrm{sl}}^{\mathrm{o}}\right)$ by rotating the electric spinor dipoles: $\operatorname{rot} \mathbf{J}_{\mathrm{e}(\mathrm{sl})}^{\mathrm{o}} \rightarrow \operatorname{rot} \mathbf{E}_{\mathrm{sl}}^{\mathrm{o}}$, where $\mathbf{E}_{\mathrm{sl}}^{\mathrm{o}}$ represents a strength of the vortex field of electric bispinor. The vector of strength of the vortex electric field $\mathbf{E}_{\mathrm{sl}}^{\mathrm{o}}$ is the electric analogue of vector $\mathbf{H}_{\mathrm{sl}}^{\mathrm{o}}$ of vortex field of magnetic spinordipols, which was discussed above. The equation a process formation of the vortex fields of electric dipoles, which are described by of vortex vectors $\operatorname{rotE}_{\mathrm{sl}}^{\mathrm{o}}$ has the form: $\mathrm{k}_{2} \operatorname{rot} \mathbf{J}_{\mathrm{e}(\mathrm{sl})}^{\mathrm{o}}=\operatorname{rot} \mathbf{E}_{\mathrm{sl}}^{\mathrm{o}}$.

The scheme of this process is given in Figure 3. Here show the electric bispinor (the electron and antielectron, respectively, with $\mathrm{e}^{-}$and $\mathrm{e}^{+}$), which is polarized under influence of the external vortex electric field described by the vortex vector rotE $\mathrm{E}^{\circ}$. When rotation of such a dipole what is carried out by rotating poles $\mathrm{N}$ and $\mathrm{S}$ of the source of vortex electric field $\operatorname{rotE}^{\circ}$, around superconductor is implemented of bispinor vortex field which described by vortex vector $\operatorname{rotE^{\circ }}{ }_{\mathrm{sl}}^{\mathrm{o}}$. The vector $\mathbf{E}_{\mathrm{sl}}^{\mathrm{o}}$ in Figure 3 is determines strength of internal vortex field of the rotating electric dipole.

It is the latter field forms the magnetomotive force, which creates a current of the magnetic charges $J_{\mathrm{g}}$ in the superconductor. The equation of the last process, as a result of which the current of magnetic charges is realized, has the form: $\mathrm{k}_{3} \operatorname{rot} \mathbf{E}_{\mathrm{sl}}^{\mathrm{o}}=\mathbf{J}_{\mathrm{g}}$. It is important to note that the vector $\mathbf{E}_{\mathrm{sl}}^{\mathrm{o}}$ is a vector of the instantaneous strength of the vortex electric field, i.e. the internal field of the ro- 
tating electric bispinor and, is generally, it not equal to the strength of the electric field $\mathbf{E}_{\mathrm{bs}}$.

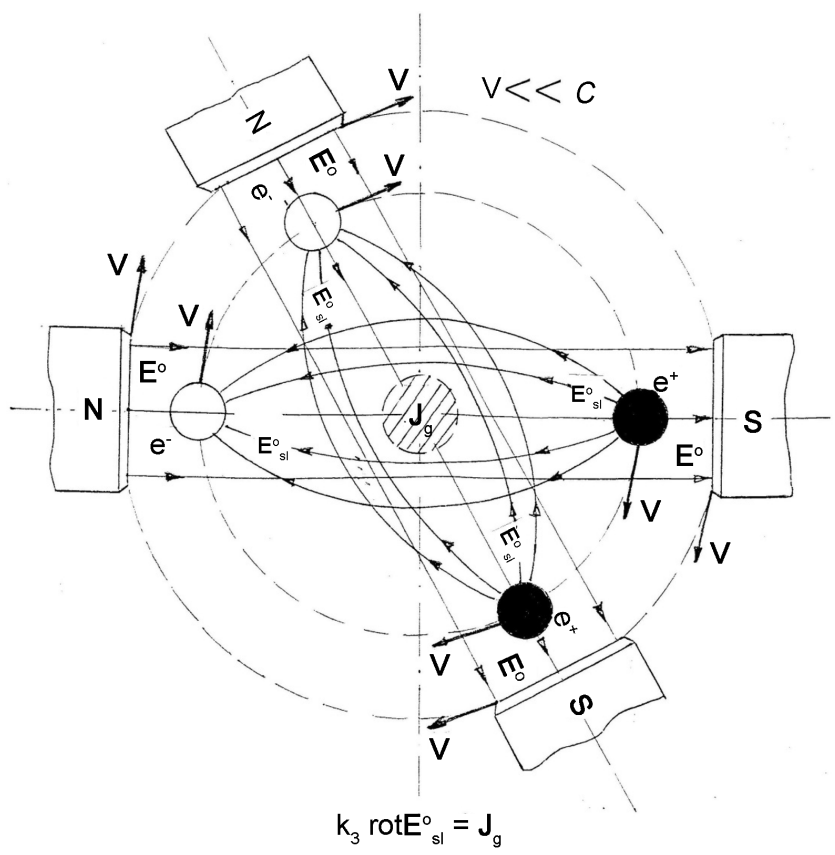

Figure 3. Scheme formation of the vortex field of electric spinor dipole $\left(\operatorname{rotE}_{\mathrm{sl}}^{\mathrm{o}}\right.$ ) by means an external rotating vortex field $\operatorname{rot} \mathrm{E}^{\circ}$ (poles $\mathrm{N}$ and $\mathrm{S}$ ).

It is easy to notice that a spinor fields which are described by vortex vectors $\operatorname{rotH}^{\circ}$ and $\operatorname{rotH}_{\mathrm{sl}}^{\circ}$ (as well as by vectors $\operatorname{rot}^{\circ}$ and $\operatorname{rotE}_{\mathrm{sl}}^{\circ}$ ) are of the vortex bispinor fields, which are generated by rotating polarized bispinorsor spinor dipoles. However, in the formation of the magnetomotive and electromotive forces are involved exclusively the fields $\operatorname{rotH}_{\mathrm{sl}}^{\mathrm{o}}$ and $\operatorname{rotE}_{\mathrm{s}}^{\mathrm{o}}$, i.e. so-called Slow vortex bispinor fields.

In order to understand this problem, it should be taken into account that spinor fields, noted above, of differ speed parameters of rotational motion of spinor dipoles. For example, the vortex magnetic field $\operatorname{rotH}^{\circ}$, as we know, is formed under of influence of a constant electric current in the conductor. As a result of the ultrahigh angular velocity when a rotation of the magnetic bispinors in this case, the magnetic field goes from the region of the space enclosed between the particles of bispinor and takes the form of the well-known magnetic field which is formed around of conductor with constant electric current (see Figure 2(a)).

As for of vortex field $\operatorname{rotH}^{\circ}{ }_{\mathrm{sl}}$ it can be created by rotating of magnet poles $\mathrm{N}$ and $\mathrm{S}$ around conductor or by rotating the conductive frame in a magnetic field. The last field is formed in conditions of slow rotation of bispinors, as the speed of their rotation is limited by possible speed revolutions of the electromotor. The circumferential velocities of the particles of spinor dipoles, which form the above-noted bispinor fields the $\operatorname{rotH}^{\circ}$ and $\operatorname{rotH}_{\mathrm{s}}^{\mathrm{o}}$, differ as minimum in $10^{4}$ times. 
All of the above, regarding the vortex fields of magnetic bispinors (magnetic spinor dipoles), directly applies to a fields of electric bispinors, defined by vortex

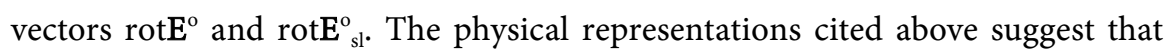
the values of the electromotive and magnetomotive forces should depend on the speed of rotation of the frame or poles. So, in theory, at the circumferential velocities $\mathrm{V} \equiv \mathrm{C}$ of the charges rotating in compositions of spinor dipoles, these forces are likely to be zero.

\section{The Reasons, Which Detained Recognition of Real Magnetic Charges, As Well as True Antielectrons in Physical Science}

There arise a natural question, how it turned out that the magnetons, and in general, magnetic spinor particles which, in author's opinion, are present in the physical masses (nucleons, atoms, substances and so on) approximately in the same amount that and the Electric particles, could remain undetected during more than one hundred years?

There are several reasons of this.

1) At first stages of researches, the superficial interpretation of results of Oersted's experiment took place when only outside and apparent participants of the experimental process, i.e. electric current and magnetic field around the conductor were considered. This superficial interpretation was adopted by Maxwell, who had her as a basis for his electromagnetic theory ignoring the popular wisdom that "the true never liese on the surface". As a result this, vicious Maxwell's electromagnetic theory had fixed on more than one hundred years an erroneous view that magnetism is an emanation of electricity. Exactly the vicious electromagnetic concept of Maxwell remained that supporting point on which was based interpretations of various magnetic and electromagnetic effects, i.e. in theoretical developments considered only electric charges under total ignoring of magnetic charges really existing in atoms and substance.

Of course, a magnetic field around a conductor with constant electric current is a real experimental fact (the experiment of Oersted). However, is not a fact, that this field is directly produced by moving electrons. As noted above, the basis for ignoring the real magnetic charges in the structures of substance was the physicists' perverse confidence, that only electrons are involved in the processes related to of realization of the magnetic field around the conductor with electric current. It is from this that and the superficial interpretation by Maxwell of the results of Oersted Experience and the centenary adherence to this interpretation by the world physical science follows. And if all decided that this is so, then what kind of magnetic charges can be? But for example, with the same "success" one can draw a conclusion that exactly the electric current flowing from electric net to a hair drier pushed air out it nozzle, if one doesn't know anything about existence of the fan and electromotor in the fan body and doesn't hear its noise and vibrations. 
There is no doubt that if the vortices of magnetic charges, organized in the conductor under action of the current electrons field could create noise and vibration Maxwell would make a quite different conclusion about the true source of the magnetic field.

2) The second and the main reason is the differentiation of the physical of bonds of the electrons and magnetic particles in the compositions of the physical masses (in atoms, substances and so on) what and identify character of the confinement of magnetic spinor particles in of solids with electric and magnetic charges [5] [8].

Basing on his own experiments and also on the results of other researches the author has discovered that magnetons (as and antimagnetons) can't be torn out of the substances by pumping it with energy. At increase of internal energy of the body (target) stronger the bonds (hardening of confinement) between magnetic charges and substance.

The notion the free particles itself in relation to electrical and magnetic spinors are significantly different. When an electric spinor, for example an electron, can be relatively easily extracted from the substance and forced to fly freely into space, magnetonscan't to leave the substance. The essence "freedom" of the magnetic particles consists in the fact that they don't interact with electric particles inside the substance at any particular moment of time. Free condition of magnetic poles is achieved by deep cooling of the substance (superconducting state). It's important to underline that only a part of magnetic spinor particles occupying the so called a potential zones of conductivety can go into such a free state. It follows from the above given considerations that unlike electric spinorsit's impossible to extract an individual magnetic particle from the substance and to force it to fly through the space (outside a physical mass).

Let us explain the latter statement.

On the Figure 4 it is a scheme of location of magneton's and electron's levels in the condensed substance state is presented on the scale of energy. It's well known that the electronic levels are in a potential pit, so for exit of an electron into the state free from links with a substance it's necessary to supply additional energy to it that is to warm it up. As for of the magnetic charges concerned, their energy levels are under a so called potential cupola, and for transition of these particles into a free state the substance should be cooled that is i.e. its internal energy should be decreased.

At the deep stages of cooling there arises the superconductivity effect what probably is an evidence of transition of magnetic spinor particles into a state free from bonds with the lattice of substance. At that electric current passes through superconductor practically without resistance, since free magnetic charges, rotating around the current lines don't has "friction" about the lattice of conductor. Any energy pumping in substance (irradiation, heating and so on), pushes magnetons on the higher bound energy levels. 


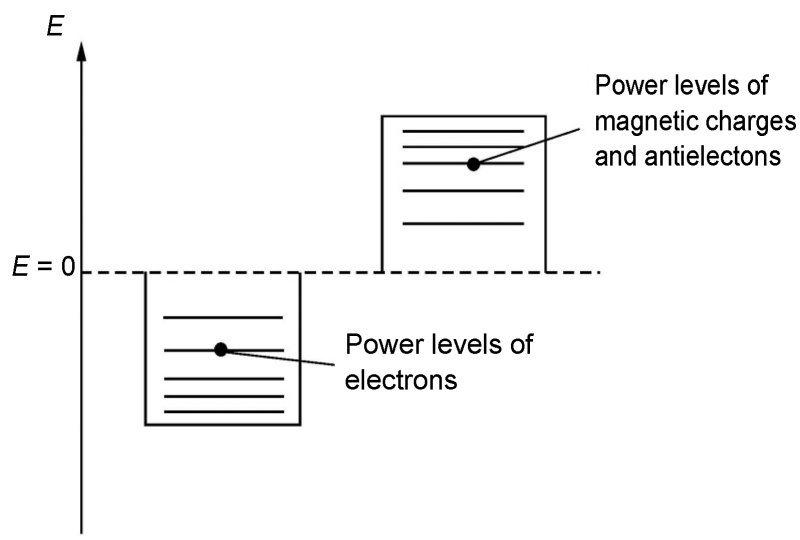

Figure 4. The disposition scheme of levels: of electrons (in the energy "pit") and magnetic charges (under the energy "cupola") on the energy scale. So, for an electron exit in a free state from bond with a substance, it is necessary to increase its internal energy, for example, to heat its. The free condition of the magnetic spinorial particles is reached at deep cooling of substance, for example, in the condition of superconductivity. General physical conditions of confinement of spinor particles and magnetic charges in particular within substance composition were considered in publications of the author (see [5] [6]) and the most detailed analysis was given in [13].

3) To the above mentioned causes, still one, i.e. subjective reason should be added. In the beginning of the XX-th century (1910), when F. Ehrenhaft began publishing the results of their experiments relating to the discovery and investigation of magnetic charges [12], the problem of these particles already wasn't considered as sufficiently important and attractive by the majority of theoreticians. The physical theory, after she "have swallowed" the fallacious "virus" of Maxwell (the definition of the erroneous Maxwell electromagnetic concept, which is given by the author of the present article), intensively developed of the global electric conception, which have "buried" not only the results Ehrenhaft's investigations, but also and interest of theoreticians to the magnetic charges.

In 1931 P. Dirac has paid attention to this problem [18]. But as it was shown in the publications of the author this "shot" of the Great theoretician finally turned out to be "the blank" since a giant charge of the Dirac's monopole (the minimum value-68.5 e) excluded any possibilities of participation of this monopoles in the structures of physical masses (atoms, substances et al.) [6]. For convinced "electricians" the real magnetic charges turned out to be not only redundant but also in some sense harmful, since their existence meant the collapse of the "castles in the air" built without their participation with the help of the virtual theoretical constructions.

In other words: so many all things are heaped in Physics without of magnetic charges, and now what to do with all this?

\section{Conclusions}

The neglect by physical science for nearly 200 years (since the discovery of Oersted in 1820) of the existence of magnetic spinor particles (magnetic charges), as well as of real antielectrons in the structures of the physical mass (in 
atoms, nucleons, and other), induced great harm to the development of the natural sciences, as well as have shut off approaches to the practice of many useful physical effects.For example, in the absence of real magnetic charges is not possible, even in the idea, is consider such of the important technical effect as the magnetoelectric induction.

"The lack of" magnetic charges and true antielectrons in the physical representations forced theorists to look for a suitable replacements (theoretical "prostheses"), which can help they to explain many magnetic and electromagnetic phenomena observed experimentally. Namely, such of prostheses are the magnetic moments, which helped "to close" in physical theory such of the serious theoretical problem, as the true nature of magnetism. In addition, in the author's publications (see [6] [15]), it is shown that namely such theoretical prostheses are the Dirac's "holes" and his famous magnetic monopole [18].

According to the author, to theoretical prostheses should be attributed and are proposed in the publications [19] [20] the collective magnetic monopoles, which as supposed, are responsible for all the magnetic manifestations in the some compounds with the structure of the spin ice. Feature in the description of the last magnetism is that the magnetic field in of the "spin ice" is created not by the electric current as in Maxwell's concept, but only by means of electron spins. In other words, the translational movement of the electrons under the formation of the magnetic field was replaced on their rotational motion.

However the greatest harm of development of natural sciences and technology haveinflict invention and introduction in the notions of model the purely electronic shell of atoms.

As a result of many years of research, the author came to the conclusion that the shells of atoms are electromagnetic and are formed by electric and magnetic bispinors or, more precisely, by the corresponding spinor dipoles. In other words, in the compositions of atomic shells, in addition to electrons, there are three more real spinor particles: true antielectrons, which are not positrons, and also, the magnetic bispinors, i.e. magnetons and antimagnetons. It is also important to understand that all these four spinor particles are in atoms and substance in the compositions of bispinors. The state of the bispinor is the ground state of the spinor particles, and the violation of this state leads to the ionization of atoms and substance.

Of course, the model of the electron shell of the atoms is the grand theoretical "puncture", but it must be remembered that the first place on the harmfulness in the physics is necessary all the same to give C.J. Maxwell and his of fallacious electromagnetic concept [7]. Namely vicious EM-concept of Maxwell, which is called in the publication author as "vicious virus Maxwell's", can to associate with the "cancerous" education in "body" of Physical science, the "metastasis" of which strike the many directions of physical theory, giving them a false and even mystical significance. Among such directions, other than those listed above may be mentioned, for example, such of resonance theoretical constructs as global expand Universe, very big explosion and a big break, the field and the 
Higgs boson, the representation of the gravitational field in the form of a curved four-dimensional space, and many, a lot others.

Common sense, according to the author, is the act of the last hope in the recognition of real magnetic charges, since of the experimental evidence of the existence of these fundamental particles in atoms and substance, by now, have accumulate more than enough.

Note 4. Base provisions of the General Physics with real magnetic charges, developed by the author, were presented at the International PIERS 2009 Moscow Conference (Progress in Electromagnetics Research Symposium) [21] [22].

\section{Acknowledgements}

The author is Grateful to technical specialists: Davydov A.A., Koshelev N.V., Lomakin A.I., Moiseenkov E.V. and Staritsin S.E. which provide technical support for the main part of his experiments, concerned the researches of magnetic charges. The author is grateful to the Editorial Board of the Journal of Modern Physics for an exceptionally friendly and super-tolerant attitude to his articles.

\section{References}

[1] Sizov, R.A. (2001) New Presentation of Nature Magnetism, Gravitation and Nuclear Forces of Bonding. Akademizdat Center "Science", Moscow.

[2] Sizov, R.A. (1971) Solid State Physics, 13, 2081-2088.

[3] Sizov, R.A. (1971) Journal of Experimental and Theoretical Physics, 60, 1363-1370.

[4] Sizov, R.A. (2005) Magnetic Elementary Particles as Stable Structural Components of Atoms and Substance. Akademizdat Center "Science", Moscow.

[5] Sizov, R.A. (2008) Electric and Magnetic Spinor Particles as Structure-Forming Components of Mass and Electromagnetic Source Gravitation. Akademizdat Center "Science", Moscow.

[6] Sizov, R.A. (2015) Journal of Modern Physics, 6, 1013-1022.

[7] Maxwell, J.C. (1873) Treatise on Electricity and Magnetism. Vol. 1-2, Clarendon Press, Oxford.

[8] Sizov, R.A. (2012) Levitation as a Spread Force Manifestation of Natural Ferrogravitation. Akademizdat Center "Science”, Moscow.

[9] Sizov, R.A. (2015) Journal of Modern Physics, 6, 1591-1601.

[10] Sizov, R.A. (2013) Transference of People and Loads in the Terrestrial Space by Means of Technical Ferrogravitation. Akademizdat Center "Science", Moscow.

[11] Sizov, R.A. (2013) Generation and Technical Application of Ferrogravitation (The Collection of the Invention of the Author in Area the Gravitophysical and Gravitochemical Technologies). Akademizdat Center "Science", Moscow.

[12] Ehrenhaft, F. (1942) Journal of the Franklin Institute, 233, 235-244.

[13] Sizov, R.A. (2011/2012) Matter, Antimatter and Energo-Medium-Physical Triad of the Real World. Akademizdat Center "Science", Moscow.

[14] Sizov, R.A. (2016) Journal of Modern Physics, 7, 2374-2397.

[15] Sizov, R.A. (2015) Journal of Modern Physics, 6, 2280-2289. https://doi.org/10.4236/jmp.2015.615232 
[16] Sizov, R.A. (2016) Journal of Modern Physics, 7, 558-572.

[17] Dirac, P.A.M. (1930) Proceedings of the Royal Society A, 126, 360.

[18] Dirac, P.A.M. (1931) Proceedings of the Royal Society A, 133, 60-72. https://doi.org/10.1098/rspa.1931.0130

[19] Castelnovo, C., Moessner, R. and Sondhi, S.L. (2008) Nature, 451, 42-45.

[20] Paulsen, C., Jackson, M.J., ELhotel, E., Canals, B., Prabhakaran, D., Matsuhira, K., Giblin, S.R. and Bramwell, S.T. (2014) Nature Physics, 10, 135-139.

[21] Sizov, R.A. (2009) Magnetic Particles (Magnetons)-Structural Components of Atoms and Substance, Immediate Sources of Magnetic Field. Theory and Experiments the Report at PIERS, Moscow.

[22] Sizov, R.A. (2009) Electric and Magnetic Spinor Particles-The Electromagnetic Source of Gravitation. Theory and Experiments the Report at PIERS, Moscow. 


\section{Appendix}

Main physical parameters of magnetic spinor particles forming together with electrical particles the atomic shells.

Charge: The magneton (magnetic spinor) and the antimgneton (magnetic antispinor) have a magnetic charges $g$ the value of which is equal to the value of the electron charge $(g=e)$. Researches of the author showed that the greatest possibility charge of a fundamental magnetic spinor particle is equal to electron charge, i.e. $\mathrm{g}_{\max }=\mathrm{e}$.

Sign of the charges: Like an electron a magneton has a charged with the sign "minus" $\left(\mathrm{g}^{-}\right)$. Sign charge antimagneton-"plus" $\left(\mathrm{g}^{+}\right)$.

Mass: Like an electron a magneton, antimagneton and antielectrons are the massless particles, because the physical mass is result of joint structural "activity" ofthe electric and magnetic spinor particles.

Class of elementary particles: The magneton is a lepton and an antimagneton-an antilepton.

Statistical properties: The magneton is a fermion (spin equal 1/2) and the antimagneton-an antifermion (spin equal -1/2).

\section{Scientific Research Publishing}

Submit or recommend next manuscript to SCIRP and we will provide best service for you:

Accepting pre-submission inquiries through Email, Facebook, LinkedIn, Twitter, etc. A wide selection of journals (inclusive of 9 subjects, more than 200 journals) Providing 24-hour high-quality service User-friendly online submission system Fair and swift peer-review system Efficient typesetting and proofreading procedure Display of the result of downloads and visits, as well as the number of cited articles Maximum dissemination of your research work

Submit your manuscript at: http://papersubmission.scirp.org/

Or contact jmp@scirp.org 